\title{
40-GHz subharmonic optical clock recovery using an injection-locked optoelectronic oscillator
}

\author{
Hidemi Tsuchida ${ }^{\text {a) }}$ \\ National Institute of Advanced Industrial Science and Technology \\ 1-1-1 Umezono, Tsukuba-shi, Ibaraki 305-8568, Japan \\ a)h-tsuchida@aist.go.jp
}

\begin{abstract}
GHz subharmonic optical clock recovery has been demonstrated using an optoelectronic oscillator (OEO) injection locked by $160-\mathrm{Gb} / \mathrm{s}$ data streams. The data streams are coupled to a photodetector in the OEO feedback loop after transmitted through a $\mathrm{LiNbO}_{3}$ intensity modulator, which enhances the residual subharmonic clock component in the data streams. The pulse width and root-mean-square timing jitter of the recovered clocks are $9.03 \mathrm{ps}$ and $221 \mathrm{fs}(10 \mathrm{~Hz}-$ $40 \mathrm{MHz}$ ), respectively.
\end{abstract}

Keywords: clock recovery, optoelectronic oscillator, injection locking, optical time division multiplexing, phase noise

Classification: Photonics devices, circuits, and systems

\section{References}

[1] J. Lasri, P. Devgan, R. Tang, and P. Kumar, "Ultralow timing jitter 40$\mathrm{Gb} / \mathrm{s}$ clock recovery using a self-starting optoelectronic oscillator," IEEE Photon. Technol. Lett., vol. 16, pp. 263-265, Jan. 2004.

[2] H. Tsuchida and M. Suzuki, "40-Gb/s clock recovery using an injectionlocked optoelectronic oscillator," IEEE Photon. Technol. Lett., vol. 17, pp. 2611-213, Jan. 2005.

[3] T. Ohno, K. Sato, T. Shimizu, T. Furuta, and H. Ito, "Recovery of $40 \mathrm{GHz}$ optical clock from $160 \mathrm{~Gb} / \mathrm{s}$ data using regeneratively modelocked semiconductor laser," Electron. Lett., vol. 39, pp. 453-455, March 2004.

[4] T. Ohno, K. Sato, R. Iga, Y. Kondo, K. Yoshino, T. Furuta, K. Yoshino, and H. Ito, "Recovery of $80 \mathrm{GHz}$ optical clock from $160 \mathrm{~Gb} / \mathrm{s}$ data stream using regeneratively modelocked laser diode," Electron. Lett., vol. 39, pp. 1398-1400, Sept. 2003.

[5] E. S. Awad, P. S. Cho, N. Moulton, and J. Goldhar, "Subharmonic optical clock recovery from $160 \mathrm{~Gb} / \mathrm{s}$ using time-dependent loss saturation inside a single electroabsorption modulator," IEEE Photon. Technol. Lett., vol. 15, pp. 1764-1766, Dec. 2003.

[6] J. H. Lee, S. Ohara, T. Nagashima, T. Hasegawa, N. Sugimoto, K. Igarashi, K. Katoh, and K. Kikuchi, "Clock recovery and demultiplexing of high-speed OTDM signal through combined use of Bismuth Oxide nonlinear fiber and Erbium-doped Bismuth Oxide fiber," IEEE Photon. Technol. Lett., vol. 17, pp. 2658-2660, Dec. 2005. 
[7] L. Hu, Y. Dong, C. Lou, and Y. Gao, "Clock extraction using an optoelectronic oscillator from high-speed NRZ signal and NRZ-to-RZ format transformation," IEEE Photon. Technol. Lett., vol. 15, pp. 981-983, July 2003.

[8] Z. Hu, H. Chou, J. E. Bowers and D. J. Blumenthal, "40-Gb/s optical clock recovery using a compact traveling-wave electroabsorption modulator-based ring oscillator," IEEE Photon. Technol. Lett., vol. 16, pp. 1376-1378, May 2004.

[9] H. Tsuchida, "Timing noise measurement of $320 \mathrm{GHz}$ optical pulses using an improved optoelectronic harmonic mixer," Opt. Lett., vol. 31, pp. 628630, March 2006.

[10] H. Tsuchida, "160-Gb/s optical clock recovery using a regeneratively mode-locked laser diode," to be published in IEEE Photon. Technol. Lett.

\section{Introduction}

All-optical clock recovery is one of the key technologies for realizing optical time-division-multiplexed (OTDM) networks that require synchronous operations such as demultiplexing, add-drop and 3R (reamplification, retiming, and reshaping) regeneration. Subharmonic clock recovery is indispensable for demultiplexing and add-drop operations and was demonstrated using optoelectronic oscillator (OEO) [1, 2], mode-locked lasers [3, 4], and phase-locked loops $[5,6]$. OEO-based clock recovery $[1,2,7,8]$ is advantageous in simultaneously extracting both electrical and optical clocks with high spectral purity. In a previous work [2] we demonstrated $40-\mathrm{GHz}$ subharmonic optical clock recovery, in which $160-\mathrm{Gb} / \mathrm{s}$ data streams were directly coupled to a photodetector $(\mathrm{PD})$ in the OEO feedback loop. The mechanism responsible for subharmonic clock recovery was the residual 40-GHz component in the 160$\mathrm{Gb} / \mathrm{s}$ data streams, which was produced by the imperfection of the OTDM multiplexer. The pulse width and root-mean-square (rms) timing jitter of the recovered clocks were $11.3 \mathrm{ps}$ and $782 \mathrm{fs}(10 \mathrm{~Hz}-18.6 \mathrm{MHz})$, respectively, which was limited by the small amplitude of the residual subharmonic component.

This paper describes improvements in the performance of subharmonic optical clock recovery at $40 \mathrm{GHz}$ using a modified OEO configuration. 160$\mathrm{Gb} / \mathrm{s}$ data streams are coupled to the $\mathrm{PD}$ after transmitted through a $\mathrm{LiNbO}_{3}$ intensity modulator in the OEO feedback loop, which enhances the amplitude of the subharmonic clock component. This modification results in shorter pulse width and smaller timing jitter as well as the more than $10 \mathrm{~dB}$ reduction in the power of the injected data streams.

\section{Experimental Setup}

Figure 1 (a) shows schematic diagram of the OEO for 40-GHz subharmonic clock recovery used in the previous work [2]. The OEO is comprised of a continuous-wave $(\mathrm{CW})$ laser, $\mathrm{LiNbO}_{3}$ intensity modulator, variable optical 


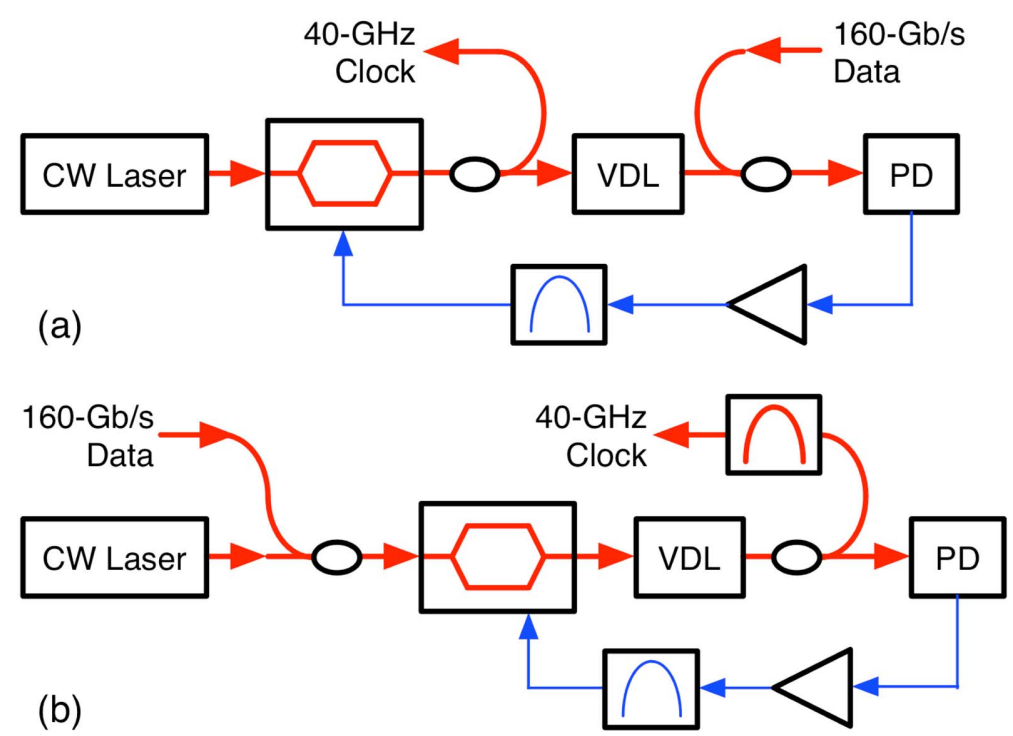

Fig. 1. Schematic diagram of optoelectronic oscillators for subharmonic optical clock recovery used in (a) previous and (b) present works.

delay line (VDL), PD, electrical amplifier, and high-Q electrical bandpass filter (BPF). The output light from the $\mathrm{CW}$ laser is input to the modulator and is converted to an electrical signal by the PD. The PD output signal is fed back to the modulator through the amplifier and BPF. The OEO is designed to oscillate around $40 \mathrm{GHz}$ and it is assumed that $160-\mathrm{Gb} / \mathrm{s}$ data streams contain subharmonic component at $40 \mathrm{GHz}$. Recovered optical clocks are extracted just after the modulator, while $160-\mathrm{Gb} / \mathrm{s}$ data streams are directly coupled to the $\mathrm{PD}$, which is advantageous for achieving wavelengthindependent and polarization-insensitive operation [2].

In the absence of injected data streams, the OEO supports self-sustained oscillation at the frequency determined by the BPF and transit time of the feedback loop. The oscillation frequency can be tuned by adjusting the VDL. With data streams injection, the OEO is forced to synchronize with the subharmonic component in the data streams. Therefore, the performance of clock recovery strongly depends on the amplitude of the subharmonic components, which was a main limiting factor in the previous work [2].

To improve the performance of clock recovery a modified OEO shown in Fig. 1 (b) is used in the present work. 160-Gb/s data streams are input to the modulator together with the CW light and are modulated by the 40$\mathrm{GHz}$ electrical clock signal, which is expected to enhance the amplitude of the subharmonic component. $40-\mathrm{GHz}$ optical clocks are extracted just after the VDL using a directional coupler and optical BPF. Although this configuration sacrifices the merit of wavelength-independent and polarization-insensitive operation, it drastically improves the performance as will be shown below.

159.25248-Gb/s data streams are generated from an actively mode-locked fiber laser (AML-FL) with a repetition frequency of $9.95328 \mathrm{GHz}$. The pulse width and center wavelength of the AML-FL output are $2.0 \mathrm{ps}$ and $1550.3 \mathrm{~nm}$, respectively. The AML-FL output pulses are encoded by a $2^{7}-1$ psuedoran- 
dom bit sequence (PRBS) pattern using a $\mathrm{LiNbO}_{3}$ intensity modulator, and are multiplexed into 159.25248-Gb/s data streams. The four-stage fiber-optic OTDM multiplexer has the delays of $6.4,3.2,1.6$ and $0.8 \mathrm{~ns}$, respectively, which ensures a PRBS pattern at the output. Although an ideal 160-Gb/s data stream contains no subharmonic components, imperfection in the multiplexer produces residual $40-\mathrm{GHz}$ tone, which enables injection-locking at the subharmonic frequency $[1,2]$.

As shown in Fig. 1(b), the 1560.0-nm CW light and data streams are combined and detected by the PD after transmitted through the modulator. The center frequency and Q factor of the BPF are $39.81291 \mathrm{GHz}$ and 961, respectively. The transit time of the feedback loop is $20.0 \mathrm{~ns}$ corresponding to the free spectral range of $50 \mathrm{MHz}$, which ensures single-mode oscillation. To evaluate the performances, optical clock waveforms are observed with an optical sampling oscilloscope (Yokogawa, AQ7750) whose trigger signal is derived from a frequency synthesizer used to mode-lock the AML-FL. Phase noise of the optical clocks is measured using a signal source analyzer (Agilent, E5052A) after electrical down-conversion [9].

\section{Results}

By adjusting the VDL the OEO oscillation frequency can be continuously tuned between 39.8008 and $39.8382 \mathrm{GHz}$ when the average power of the $\mathrm{CW}$ light incident upon the $\mathrm{PD}$ is $-7.77 \mathrm{dBm}$. Injection locking is achieved for the OEO frequency detuning between $-0.55 \mathrm{MHz}$ and $0.19 \mathrm{MHz}$ from the nominal clock frequency of $39.81312 \mathrm{GHz}$. The average powers of the $159.25248-\mathrm{Gb} / \mathrm{s}$ data streams required for achieving stable injection locking are $-7.12,-18.1$ and $-8.27 \mathrm{dBm}$ for the frequency detuning of $-0.55,-0.01$ and $0.19 \mathrm{MHz}$. respectively.

Figure 2 shows the optical sampling traces of the $159.25248-\mathrm{Gb} / \mathrm{s}$ data streams and 39.81312-GHz optical clocks. Figure 2 (a) corresponds to the input data streams. The amplitude of the residual 40-GHz clock component contained in the data streams is estimated to be $0.054 \%$ of the average intensity [10]. Figure 2(b) shows the result from the previous work representing the waveform of optical clocks obtained with the configuration shown in Fig. 1(a). The average optical powers incident upon the PD are -6.54 and $-4.12 \mathrm{dBm}$ for the $\mathrm{CW}$ light and data streams, respectively. A relatively large pulse width of $11.3 \mathrm{ps}$ is attributed to the smaller amplitude of the subharmonic clock component in the data streams.

Figure 2 (c) corresponds to the optical clocks obtained with the configuration shown in Fig. 1(b). The average powers incident upon the PD are -7.74 and $-16.1 \mathrm{dBm}$ for the CW light and data streams, respectively. The observed waveform can be well fitted to Gaussian shape with the full width at half maximum (FWHM) of 9.03 ps. The FWHM of the spectral envelope is measured to be $0.437 \mathrm{~nm}$ giving the time-bandwidth product of 0.486 . By comparing Figs. 2 (b) and (c), it can be seen that the present configuration is effective for reducing the pulse width. The improvement can be attributed 


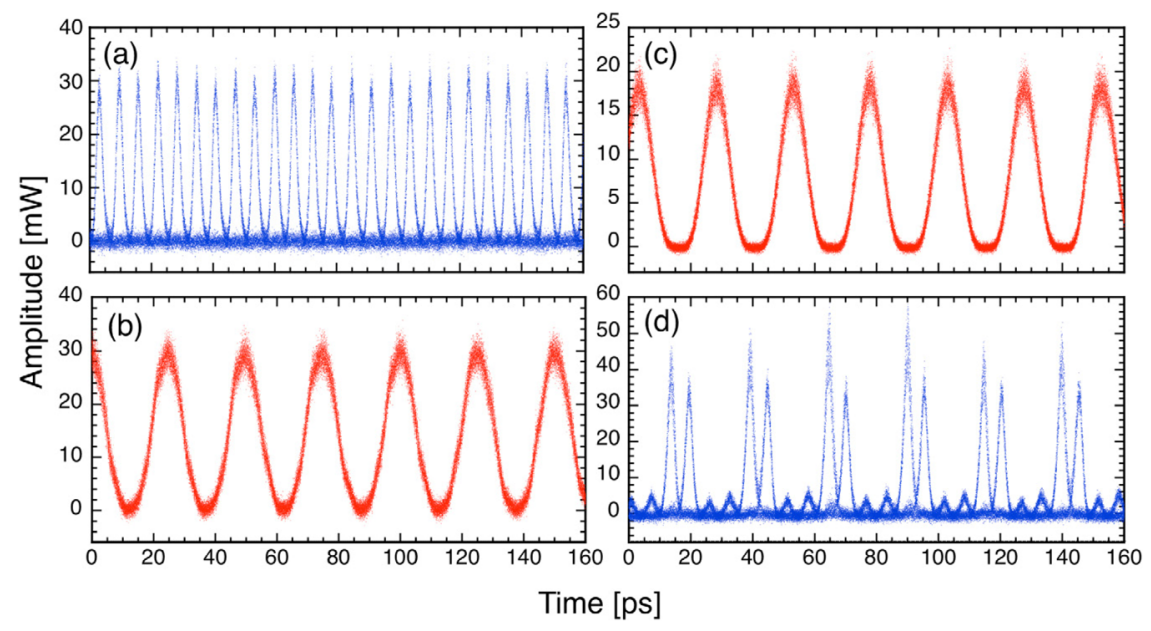

Fig. 2. Optical sampling traces: (a) $160-\mathrm{Gb} / \mathrm{s}$ input data streams, (b) 40-GHz clocks obtained in the previous work [2], (c) 40-GHz clocks obtained in the present work, and (d) 160-Gb/s data streams after transmitted through the modulator.

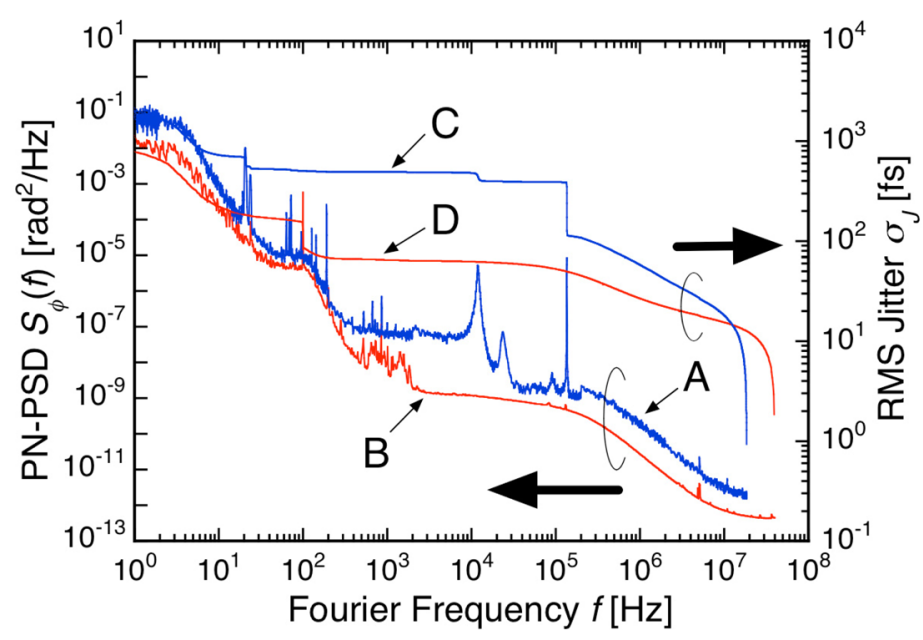

Fig. 3. PN-PSD $S_{\phi}(f)$ (curves A and B) and root-meansquare (rms) timing jitters $\sigma_{J}$ (curves $\mathrm{C}$ and $\mathrm{D}$ ) of the 39.81312-GHz optical clocks. Curves A and $\mathrm{C}$ were obtained in the previous work [2], while curves B and D represent the results obtained in this work.

to the increased amplitude of the subharmonic clock component, which is verified from the waveform of the data streams after transmitted through the modulator shown in Fig. 2 (d). It can be seen that the data streams are transformed into twin pulses with a period of $25.12 \mathrm{ps,} \mathrm{which} \mathrm{contain} \mathrm{larger}$ subharmonic clock component than those before modulation. It should be also noticed that the average power of the data streams incident upon the $\mathrm{PD}$ is reduced by $12 \mathrm{~dB}$ as compared with the previous work [2].

Figure 3 shows the phase noise power spectral density (PN-PSD) $S_{\phi}(f)$ (curves $\mathrm{A}$ and $\mathrm{B}$ ) and rms timing jitters $\sigma_{J}$ (curves $\mathrm{C}$ and $\mathrm{D}$ ) calculated by 
using the relationship

$$
\sigma_{J}=\frac{1}{2 \pi f_{\mathrm{rep}}} \sqrt{\int_{f_{l}}^{f_{h}} S_{\phi}(f) d f},
$$

where $f_{\text {rep }}=39.81312 \mathrm{GHz}$ represent the clock frequency, $f_{l}$ and $f_{h}$ denote the lower and upper limits of integration, respectively. The value of $\sigma_{J}$ is calculated for fixed $f_{h}$ and is plotted as a function of $f_{l}$. Curves $\mathrm{A}$ and $\mathrm{C}$ represent the results obtained in the previous work [2]. The rms jitter amounts to $728 \mathrm{fs}(10 \mathrm{~Hz}-18.6 \mathrm{MHz})$, which is about 2.8 times larger than that obtained in 40-Gb/s line rate clock recovery [2]. Curves B and D represent the results obtained with the configuration shown in Fig. 1(b). By comparing curves $\mathrm{A}$ and $\mathrm{B}$, it can be seen that the noise above $100-\mathrm{Hz}$ Fourier frequency is considerably reduced. The rms jitter amounts to $221 \mathrm{fs}(10 \mathrm{~Hz}-$ $40 \mathrm{MHz}$ ) corresponding to $0.88 \%$ of the nominal pulse period. These values are comparable to those obtained in $40-\mathrm{Gb} / \mathrm{s}$ line rate clock recovery [2].

\section{Summary}

In summary, 40-GHz subharmonic optical clock recovery from $160 \mathrm{~Gb} / \mathrm{s}$ data streams was demonstrated using an injection-locked OEO. To enhance the residual clock component, $160 \mathrm{~Gb} / \mathrm{s}$ data streams were coupled to the PD after transmitted through the modulator in the OEO feedback loop. The pulse width and rms timing jitter of the recovered clocks were $9.03 \mathrm{ps}$ and $221 \mathrm{fs}(10 \mathrm{~Hz}-40 \mathrm{MHz})$, respectively. The minimum average power of the data streams required for stable injection locking was $-18.1 \mathrm{dBm}$. These performances are much better than those obtained in the previous work and are comprable to the those of $40-\mathrm{GHz}$ line rate clcok recovery [2]. 